\title{
Commentary
}

\section{WHO Standard Acupuncture Point Locations}

\author{
Sabina $\operatorname{Lim}^{1,2,3}$
}

${ }^{1}$ Division of Acupuncture \& Meridian, WHO Collaborating Center for Traditional Medicine, East-West Medical Research Institute, ${ }^{2}$ Department of Applied Eastern Medicine, Graduate School and ${ }^{3}$ Department of Meridian and Acupuncture, Graduate School of Basic Eastern Medical Science, Kyung Hee University, Seoul, South Korea

\begin{abstract}
'WHO Standard Acupuncture Point Locations in the Western Pacific Region (WHO Standard) was released in 2008. Initially, there were 92/361 controversial acupuncture points (acupoints). Through seven informal consultations and four task force team meetings, 86 points were agreed upon among the 92 controversial acupoints, leaving 6 remaining controversial acupoints, demanding active research in the future. This will enhance the reproducibility and validity of acupuncture studies. It will also lead to a better understanding of acupuncture mechanisms in order to optimize its clinical efficacy for a range of diseases and syndromes. This book has two parts: General Guidelines for Acupuncture Point Locations and WHO Standard Acupuncture Point Locations. First of all, familiarity with the General Guidelines for Acupuncture Point Locations in this book can help the reader to understand and use the contents of this book in depth. I would like to thank all of the participating experts and scholars for this great work, who have overcome the limits of previous acupuncture references. I also appreciate the dedicated effort and harmonious leadership of Dr Choi Seung-hoon, former Regional Adviser in Traditional Medicine of Western Pacific Office, WHO.
\end{abstract}

Keywords: acupoint - eastern medicine - traditional medicine

As acupuncture therapy has become an important component of Complementary and Alternative Medicine throughout the world, there have been many efforts to bring scientific rigor in understanding the mechanisms behind and evidence-based efficacy of acupuncture therapy. While there is extensive literature on acupuncture in scientific journals of high standard, the discipline still suffers from a lack of verifiable standards and systematic methodology for reproducing and validating individual studies. An examination of the literature shows that the locations of acupoints and the particular methods of acupuncture stimulation are diverse among individual research reports. These problems lead to

For reprints and all correspondence: Sabina Lim, MD, PhD, Professor Department of Applied Eastern Medicine, Graduate School, Kyung Hee University, \#47 Gyeonghuidae-gil, Dongdaemun-gu, Seoul, 130-

701, Korea. Tel: 82-2-961-0324; Fax: 82-2-961-7831;

E-mail: lims@khu.ac.kr ambiguity in conclusions for any meta-analysis and result from the lack of a common protocol on acupuncture-related studies. Thus, there has been a need to standardize acupuncture point (acupoint) locations so as to form a consensus on research methods.

In October 2003, the first Informal Consultation on the Development of WHO Standard of Acupuncture Point Locations was convened. As each Member State has its own initiatives and traditions, in the beginning it seemed almost impossible to establish international standardization. Fortunately, through seven informal consultations and four task force team meetings, the experts reached a consensus on many points. In the end, 86 of the 92 controversial points (among 361 total acupoints) were standardized.

This standardization will enhance the reliability and reproducibility of acupuncture studies, which in turn will lead to a better understanding of acupuncture's mechanisms so as to ultimately optimize its clinical efficacy for a range of diseases and syndromes. Now a number of 
acupuncture-related journals will make it clear in their instructions that papers must follow this WHO Standard Acupuncture Point Locations in the Western Pacific Region (WHO Standard) (1). Therefore, researchers and practitioners are encouraged to utilize the WHO Standard to ease the review process of each journal and institutional review boards (IRB).

First of all, familiarity with the General Guidelines for Acupuncture Point Locations in this book can help the reader to understand and use the contents of this book in depth. These guidelines include essential terms and definitions, which provide a very solid and clear overview of the book. For acupoint locations, a vertical and horizontal coordinate method is adopted as often as possible and employs modern anatomical terms consistently, which helps the reader to select, find and use the exact points. In addition, the anatomical landmark method is used to provide absolute and accurate standards for locating acupoints. When it comes to cun, the standard measuring unit, this book adopts the idea of separating B-cun (Proportional bone cun) from F-cun (Finger cun) which helps in the finding and measurement of more exact locations (2). The illustrations are quite simple, but very clear and accurate and some amplified drawings are included for more exact information. The 'Notes' section offers supplementary explanations on key points, such as an acupoint's relationship with adjacent acupoints so that readers can understand their correlations and observe the differences among different individuals. Despite the fact that 355 points were successfully standardized, there still remain six controversial acupoints, which are described as alternative acupoint locations under 'Remarks' and remain an active research area in the future.

Now, based on these WHO Standard, acupuncture researchers are able to conduct their studies and also share more active and accurate scientific communications in traditional medicine. With global standards and international competitiveness, it will definitely help to solve ambiguity in conclusions for any meta-analysis and establish a common protocol in acupoint locations. In the near future, I believe we can expect more evidence-based clinical efficacy for acupuncture therapy and make Eastern medicine more accessible and valuable in human health care.
The success of this collaboration is attributed to all the experts and scholars especially from China, Japan and Korea, who have finally agreed to reach a consensus despite all the different traditions and backgrounds they have. Once again, I would like to thank all the participants for another great accomplishment, which has paved the way for a new era of acupuncture study and therapy. I also appreciate the dedicated efforts and harmonious leadership of Dr Choi Seung-hoon, former Regional Adviser (2003-08) in Traditional Medicine of Western Pacific Office, WHO. Traditional medicine in East Asia has been divided by regional groups, such as TCM (traditional Chinese medicine), Kampo medicine (Japan), TKM (traditional Korean medicine), etc. However, Dr Choi's enthusiasm for traditional medicine and insight into the future helped to integrate all of these groups and develop Eastern medicine.

Another guide book on acupoints called 'Standard Acupuncture Nomenclature' (second edition) (3) was published in 1993. This includes the standardized name of each classical point, a brief explanation of the name of the point and a multilingual comparative list of the names of the point (http://www.wpro.who.int/ publications/pub_9290611057.htm). In 2007, 'WHO International Standard Terminologies on Traditional Medicine in the Western Pacific Region' (4), which contributes to standardization and globalization of traditional medicine, was published and it is still freely accessible on the WHO Website. (http://www.wpro. who.int/NR/rdonlyres/14B298C6-518D-4C00-BE02-FC31 EADE3791/0/WHOIST_26JUNE_FINAL.pdf).

\section{References}

1. WHO Regional Office for the Western Pacific. WHO Standard Acupuncture Point Locations in the Western Pacific Region. Manila: World Health Organization, 2008.

2. Yin CS, Park HJ, Seo JC, Lim S, Koh HG. Evaluation of the cun measurement system of acupuncture point location. Am J Chin Med 2005;33:729-35.

3. WHO Regional Office for the Western Pacific. Standardized Nomenclature for Acupuncture. Manila: World Health Organization, 1993.

4. WHO Regional Office for the Western Pacific. WHO International Standard Terminologies on Traditional Medicine in the Western Pacific Region. Manila: World Health Organization, 2007.

Received December 10, 2008; accepted January 12, 2009 


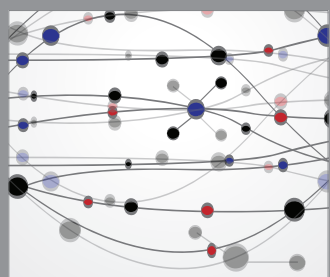

The Scientific World Journal
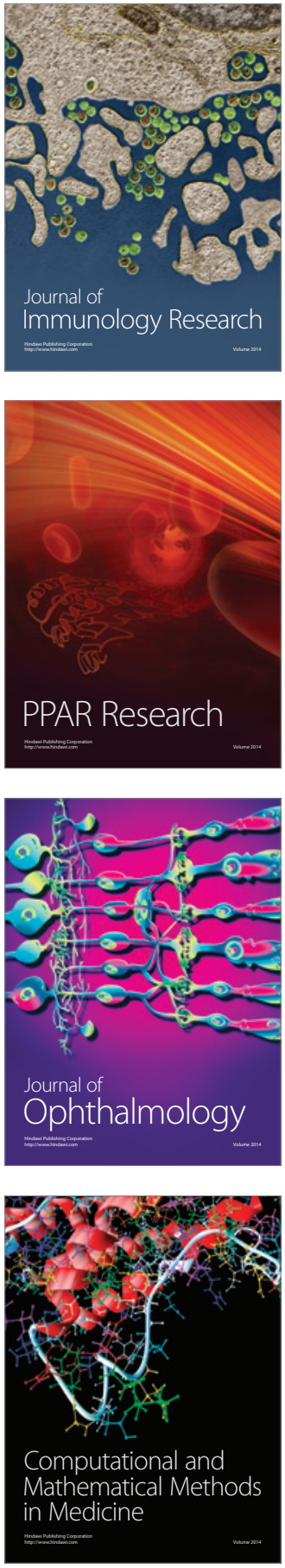

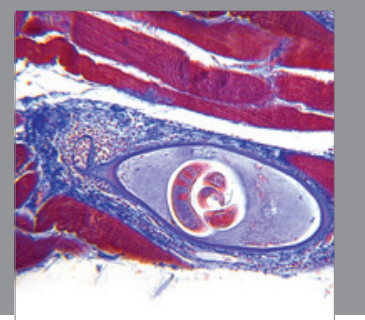

Gastroenterology

Research and Practice
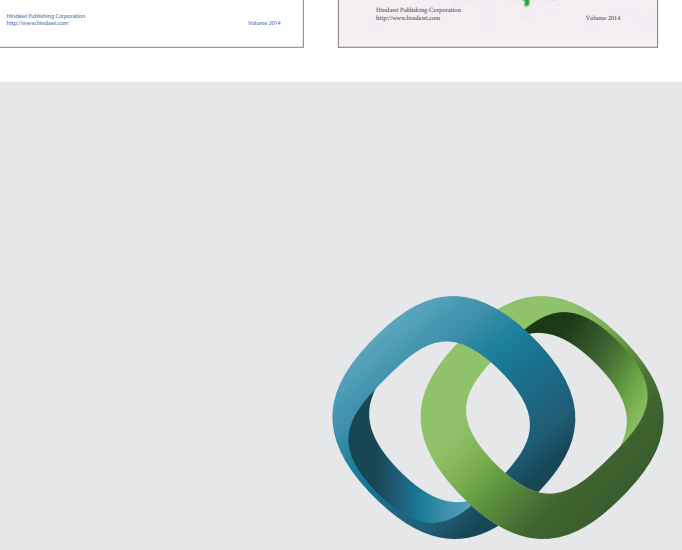

\section{Hindawi}

Submit your manuscripts at

http://www.hindawi.com
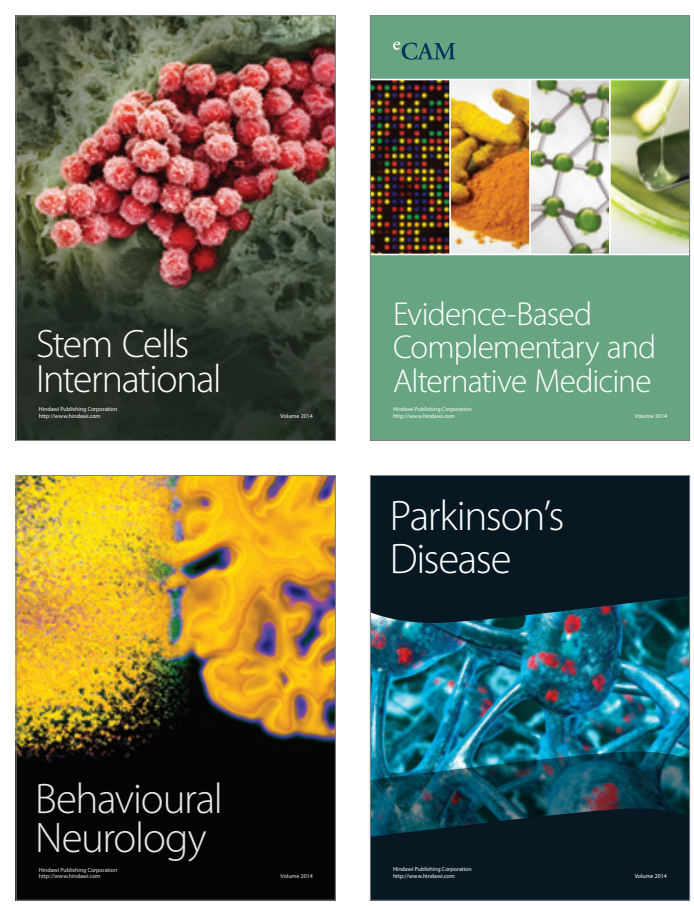

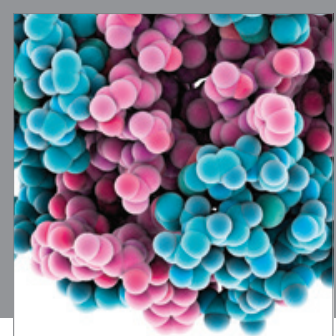

Journal of
Diabetes Research

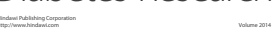

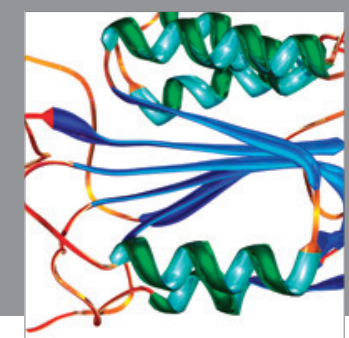

Disease Markers
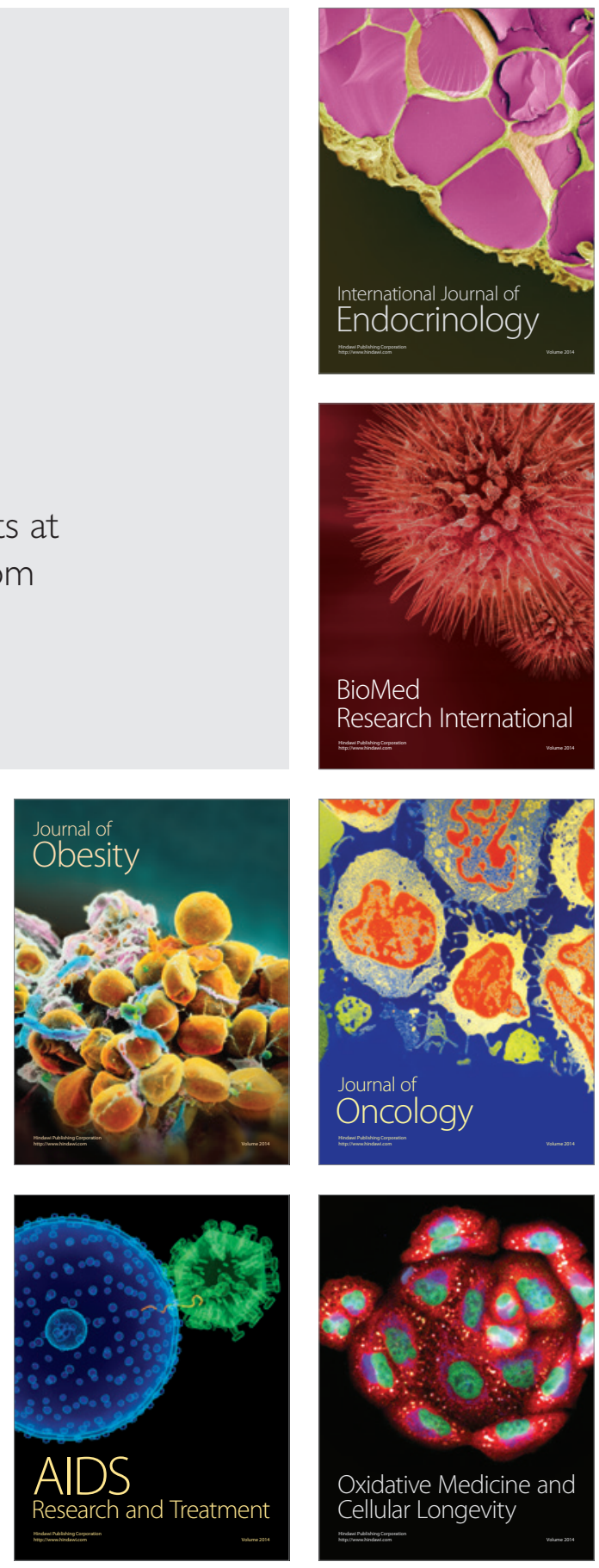